\title{
Applicability of DSM-V substance use disorder (SUD) criteria in medication overuse headache (MOH)
}

\author{
Thaiza Agostini Córdoba de Lima' ${ }^{1}$, Mario Fernando Prieto Peres ${ }^{1,2}$ (D), Stephen D Silberstein ${ }^{3}$
}

${ }^{1}$ Hospital Israelita Albert Einstein, São Paulo, SP,Brazil

${ }^{2}$ Instituto de Psiquiatria do Hospital das Clínicas da FMUSP, São Paulo, SP, Brazil

${ }^{3}$ Thomas Jefferson University: Philadelphia, PA, USA

$凶$

Mario F P Peres; Hospital Israelita Albert Einstein; Av. Albert Einstein, 627, Bloco A1, $1^{\circ}$ andar, Sala 110, Jardim Leonor; 05652-900, São Paulo-SP, Brazil. E-mail: mariop3r3s@ gmail.com

\section{Edited by}

Marcelo Moraes Valença

Keywords:

Medication Overuse Headache

Substance use disorder

Addiction

Dependence

Abuse

Migraine

\begin{abstract}
Medication overuse headache $(\mathrm{MOH})$ is a chronic secondary headache disorder attributed to the frequent or regular use of analgesics or acute antimigraine drugs in patients with a primary headache disorder. In addition, it has been linked to substance use disorder (SUD) also known as drug addiction, a persistent use of drugs or substances, despite substantial damage and adverse consequences, diagnosed by DSM-V criteria. At this time, apart from opioids, acute headache medications are not included in SUD. Despite the idea that the compulsive search for reward in $\mathrm{MOH}$ is similar to that observed in substance dependence, the DSM-V SUD criteria have never been carefully applied to $\mathrm{MOH}$. We propose to discuss each DSM-V criterion of SUD diagnosis to see whether it is appropriate to use in $\mathrm{MOH}$. We considered it was not-applicable when dealing with a situation that could be explained both by addictive behavior and by poorly controlled primary headache. We conclude that the SUD criteria should not be applied to patients who meet the criteria for $\mathrm{MOH}$.
\end{abstract}




\section{Introduction}

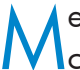
edication overuse headache $(\mathrm{MOH})$ is a common chronic secondary headache disorder attributed to the frequent or regular use of analgesics or acute antimigraine drugs in patients with a primary headache disorder. It is commonly associated with chronic migraine and chronic tension-type headache. $\mathrm{MOH}$ implies the idea of a paradoxical effect of long term, frequent use of acute medication. Although $\mathrm{MOH}$ is more likely to occur with opioids use, by definition, any abortive medication is considered to cause this condition. ${ }^{1}$ The diagnosis of $\mathrm{MOH}$ is based on the International Classification of Headache Disorders 3rd edition (ICHD-3) 8.2 MOH is defined as: headache occurring for 15 or more days/month in a patient with a pre-existing primary headache and regular overuse of acute or symptomatic headache medication for 10 (ergotamine, triptans, analgesic combinations and opioids) or 15 (simple non-opioid analgesics and NSAIDs) or more days/month, for more than 3 months. ${ }^{2}$

$\mathrm{MOH}$ has defined diagnostic criteria, but controversy still exists about its validity. ${ }^{3}$ The duration of use, quantity and type of medication needed to cause $\mathrm{MOH}$, is arbitrary in the absence of evidence. ${ }^{4}$ Furthermore, evidence of cause and effect is also weak. In population studies, the link between frequent use of medications and increased frequency of headache is well demonstrated. ${ }^{5}$ However, this causal relationship can be difficult to ascertain in individual cases. Not all patients who use analgesic medications excessively develop chronic headache. In addition, overuse of medication can be a response to chronic headaches, not the cause. ${ }^{1}$

Another controversy is the management of patients with $\mathrm{MOH}$. Elimination or reduction of overuse to less than 2 days per week ${ }^{6}$ with or without concomitant preventive medication are widely debated options ${ }^{7}$ Many use a bridging therapy to prevent withdrawal symptoms. ${ }^{8}$

It is also necessary to refine the meaning and establish the differences between the terms: use, misuse, overuse, abuse, and dependence which are not clearly defined in headache medicine. ${ }^{9}$ Only the term overuse appears in the headache classification ${ }^{2}$, but does not appear in the latest version of the Diagnostic and Statistical Manual of Mental Disorders (DSM). ${ }^{10}$

$\mathrm{MOH}$ has been linked to addictive disorders. Studies applying DSM-IV diagnostic criteria for dependence in $\mathrm{MOH}$ patients, showed $50 \%$ to $68 \%$ of dependence. 11,12 After May 2013, when DSM-V was published, significant changes in classification and criteria for substance use disorder and dependence occurred. DSM-IV abuse and dependence have been combined into one new DSM-5 diagnosis: 'Substance Use Disorder'. ${ }^{13}$

Substance use disorder (SUD) is considered a persistent use of drugs or substances, despite substantial damage and adverse consequences. Pathophysiology is associated with changes in the reward system, the brain circuits involved in pleasure, learning, stress, decision making and self-control. ${ }^{14}$ It is believed to be a subset of brain and behavioral disorders, similar to the spectrum of obsessivecompulsive disorder. ${ }^{15}$ The classes of substances include: alcohol, cannabis, hallucinogens, inhalants, opioids, sedatives, hypnotics, anxiolytics, stimulants, tobacco, and other or unknown substances. ${ }^{10}$ Apart from opioids and barbiturates, acute headache medications are not included. But compulsive reward seeking in $\mathrm{MOH}$ similar to that seen in substance dependence has been discussed. $16-18$

ICHD-3 suggests the similarity between patient's behavior with $\mathrm{MOH}$ and SUD and recommends the use of the Severity of Dependence Scale (SDS) score to predict $\mathrm{MOH}$ among headache patients. However, the criteria do not allow us to distinguish these conditions, nor to understand the complex relationship between patient behavior and medication intake. DSM-V substance Use Disorder (SUD) criteria has never been validated in $\mathrm{MOH}$ and other headache disorders. We aim analyze the SUD in order to discuss it's appropriateness in headache medicine. We also reviewed the definitions of: overuse, misuse, abuse, dependence, addiction, tolerance and withdrawal, and suggest a new classification system for patients with frequent acute headache medication use.

\section{Terms and Definitions}

In 1988 headache was associated with painkillers medications. Since then, there have been changes in nomenclature. "Drug-induced headache" was first used, then "headache induced by chronic substance use or exposure" and now, "medication overuse headache", was defined in 2004. 2,19

Although widely used, there is no clear definition of "overuse", "misuse" and "abuse", often used interchangeably. Other related terms can also be confusing, such as drug addiction, addiction, tolerance, 
and withdrawal. All these terms need to have a clear and universal meaning to avoid misunderstandings in medical practice.

The inappropriate use of those terms is not exclusivity the field of neurology. These terms are often used interchangeably in articles related to laxatives, nasal decongestants, tranquilizers, sleeping aids, analgesics for indications other than headache, and over-the-counter medications. ${ }^{20-23}$

According to recent medical literature, the definitions based on health-related use are as follows ${ }^{10,24,25}$ :

Use: Correct use of dose, administration interval and indication.

Overuse: Excessive use related to the frequency or duration of treatment. No behavior pattern of intake is attached.

Misuse: Taking the medication inappropriately, not respecting the prescription. Incorrect use of dose, administration interval and indication. For example, skipping or doubling doses or using for a purpose not consistent with legal or medical guidelines. It has a negative impact on psychological or physical health, but does not fulfill the DSM-V criteria for substance use disorder.

Abuse and Dependence: The most recent classification combines the two terms into a single unified category, named as substance use disorder (SUD). Both terms are related to the harmful use a drug or medication for a nonmedical reason, the diagnosis is based on DSM- 5 criteria which will be discussed later.

Addiction: The DSM-5 did not include the term addiction to avoid using a word with a strong stigma, to describe a disorder with a wide spectrum, as SUD can be classified as mild, moderate and severe. There is a potential negative connotation. ${ }^{26}$

Tolerance: It is a neuropharmacological phenomenon. There is a change in the dose and effect correlation. The individual needs a larger dose to obtain the desired effect or a reduced effect is obtained after consuming the usual dose. Tolerance is extremely variable depending on the individual, the substance and the delivery of the substance.

Withdrawal: It is a substance-specific syndrome that occurs when concentrations of a substance in the blood or tissues decrease in an individual with chronic use. The symptoms are class dependant; therefore, there are distinct diagnostic criteria for different classes of substances. No tolerance or withdrawal is required for the diagnosis of substance use disorder (SUD).

Intoxication: It is a substance-specific reversible syndrome due to its recent ingestion. Intoxication can be acute or chronic, symptoms vary depending on the time of exposure.

\section{SUD criteria applicability in $\mathrm{MOH}$ context}

Our proposal is to discuss each SUD DSM-V criterion in order to assess whether it is applicable or if adaptation is necessary, to chronic headache and frequent medication use. We considered the criterion as non-applicable when dealing with a situation that could be explained by both addictive behavior and poorly controlled primary headache. For example, how to differentiate whether the use of medication was motivated for pain reduction or represents dependence behavior? How to assess whether damage in professional and personal life is caused by poorly controlled primary headache or by a psychiatric disorder related to substance use?

$\mathrm{MOH}$ is associated with barbiturates, analgesic combinations, metamizole (dipyrone), acetaminofen, acetylsalicylic acid, caffeine, opioids, ergot, nonsteroidal anti-inflammatory drugs (NSAIDs), and triptans. In our analysis, the criteria applicability to opioids and barbiturate use was excluded, since OUD and barbiturates dependence are widely described conditions in the literature with diagnostic criteria well defined by the DSM-5. The applicability for analgesic combination with caffeine acting as adjuvants was considered in this study since caffeine is not listed as a substance included in SUD criteria. Caffeine is the only substance listed in DSM-V that is not included in the category for SUD.

SUD diagnosis is made when two or more criterion are present within a 12-month period. In contrast the period for the diagnostic criteria of $\mathrm{MOH}$ is 3 months.

The following is a critical analysis of the DSM-V SUD criteria in patients that fulfill criteria for MOH. DSM-5 diagnostic criteria for SUD are based on eleven topics. Each topic represents a manifestation of a problematic pattern of use leading to clinically significant impairment or distress.

Item 1. Often taken in larger amounts or over a longer period than was intended. 
In the context of chronic pain medications use, the definition of "large amounts" and "longer period" can be subjective. Is this criterion about misuse or overuse? Doubt shows how differentiating between overuse and misuse can be challenging. Our suggestion is first to identify whether it is a prescription or an over-the-counter medication. The use of doses higher than those prescribed or motivated by a situation other than indicated classifies the situation as misuse. When the patient is actually taking medication more frequently then prescribed, it should not be considered as overuse because it could be a consequence of a poor headache control.

We suggest not considering the criterion based only on a simplistic analysis of days of the months that medication was used, as it is done in the classification of $\mathrm{MOH}$.

Item 2. A persistent desire or unsuccessful efforts to cut down or control use.

This is clearly appropriate for illicit drugs and alcohol, but its applicability for excessive acute headache medication use is challenging. Since the use of an acute medication is for a medical reason and not recreation, this item should not be applicable in headache medicine.

Patients often want to decrease their medication, but if the medication has been taken to control a headache disorder, and the headache is controlled by preventive measures, acute medications are often no longer necessary. Efforts or desire to cut down or control analgesic by headache sufferers is dependent on headache control not just patients desire to not take acute medication.

Item 3. A great deal of time is spent in activities necessary to obtain, use, or recover from the substance's effects.

Analgesics are easily obtained over-the-counter, and if a prescription is needed, from medical practitioners and pharmacies. Thus, no considerable effort is necessary to obtain the medication. As in item 2, this item fits the context of drug dependence, such as opioids but not for other analgesics, or headache attack abortives. In our analysis we have also considered this criterion as non-applicable.

Item 4. Craving or a strong desire or urge to use the substance.

Patients with inadequately treated pain might exhibit drugseeking behaviour similar to that of true dependence. Opioid-based studies suggest that, to differentiate the two situations, the dose of medication should be increased.
The search behavior is resolved if the pain is controlled with a higher dose, while it remains the same or worsens in the actual addictive behavior. ${ }^{27}$ We excluded opioids from our analysis and there is no recommendation to infer the same approach to other drug classes. Furthermore, the strong desire or urge to use the medication should not be considered a pathological behavior pattern if the patient is in pain, and has a strong desire or urge to alleviate pain is perfectly legitimate, should not be considered as unhealthy.

Item 5. Recurrent use resulting in a failure to fulfill major role obligations at work, school, or home.

Recurrent use of acute headache medication may result in a failure to fulfill major role obligations, but it unrelated to addiction behaviours. Migraine burden on social and professional life is widely known, several studies have documented migraine's impact on functional ability. If acute medication is causing side effects such as nausea, drowsiness or cognitive impairment, leading to a dysfunction then it is just an undesired side effect, not addiction behaviour.

Item 6. Continued use despite having persistent or recurrent social or interpersonal problems caused or exacerbated by its effects.

Item 7. Important social, occupational, or recreational activities are given up or reduced because of use.

Both criteria 6 and 7 were analyzed simultaneously, as we consider them redundant in the current context.

According to the explanation of criterion 5, migraine impacts on interpersonal relationships and activities of social life. The burden of migraine needs to be differentiated from the consequences of the effects of medications. Thus, we consider that this criterion requires adaptation, in order to emphasize the need to seek additional effects due to the use of medications.

Item 8. Recurrent use in situations in which it is physically hazardous.

Item 9. Continued use despite knowledge of having a persistent or recurrent physical or psychological problem that is likely to have been caused or exacerbated by the substance.

Both criteria 8 and 9 were analyzed simultaneously. These criteria can be considered when the patient persist with 
the medication use even when he is aware of damage in physical and mental health and another treatment alternative have been offered. For example, continue use despite medical contraindication for presenting side effects, such as: renal failure or gastritis caused by nonsteroidal anti-inflammatory drugs or harmful sleepiness secondary to medications.

\section{Item 10. Tolerance.}

We consider this criterion as non-applicable for two main reasons. First, the tolerance and withdrawal syndrome was excluded from the DSM- 5 criteria for substance use disorder when use is prescribed and treatment is under adequate medical supervision. The justification for this consideration is that the symptoms are likely to be iatrogenic and not pathological.

Second, in order to assess the possibility of tolerance, the following situations should be excluded: change in the medication formulation or brand, drug interactions, disabsorptive disorders, and occurrence of a new disease justifying the symptoms. However, in the context of chronic pain, there is one more differential diagnosis of drug tolerance, called "pseudotolerance", in which the need for higher doses is due to the progression or exacerbation of the underlying primary headache. Therefore, tolerance criterion is not applicable in the context of $\mathrm{MOH}$.

Item 11. Withdrawal.

The fundamental requirement for withdrawal syndrome is the development of a behavior problem associated with physical symptoms due to the interruption or reduction of prolonged substance use. According to the DSM-V criteria, there are specific withdrawal symptoms for each substance, but what is common to all substances is that the symptoms must cause suffering and damage to social or professional life, and the symptoms cannot associated or explained by another medical condition. Furthermore, the diagnosis cannot be made for any substance, but only for substances with a described withdrawal syndrome. For example, there are no described withdrawal syndrome for phencyclidine and other hallucinogens, although these substances can cause dependence. Moreover, as with the tolerance criterion, the abstinence syndrome was excluded from the DSM-5 criteria for medication-prescribed use disorder.

With the above information, the question arises: Apart from opioids and caffeine, is there withdrawal syndrome described for medications that cause $\mathrm{MOH}$ ? This is a question that divides opinions between authors.

The term "withdrawal headache" has been widely used in the context of medication discontinuation in patients with $\mathrm{MOH}$. Stopping the acute medication may result in symptoms such as increase of headache, nausea, vomiting, arterial hypotension, tachycardia, insomnia and anxiety. The duration and severity of withdrawal seems to depend on the type of overused headache drug, shorter in patients overusing triptans than in patients overusing ergots or analgesics. The use of corticosteroid bridge therapy significantly reduced the duration and intensity of these symptoms, as the use of rescue medications. There is debate as to whether headache rebound and associated symptoms are drug-related withdrawal syndrome or whether it occurs due to worsening primary headache. Thus, we consider that this criterion is not applicable until further studies clarify the pathophysiology of these symptoms.

In summary, all of the eleven criteria for SUD need adaptation or have no applicability in $\mathrm{MOH}$. Therefore, we conclude that the SUD criteria should not be applied to the patient who meets the criteria for $\mathrm{MOH}$.

\section{Conclusion}

DSM-V SUD criteria is not applicable in the context of acute headache medication other than opioids and barbiturates. ICHD-3 MOH criteria do not adequately address the spectrum of addiction. Therefore, the criteria are not an appropriate diagnosis method in this subject. Studies are needed to develop and validate new diagnostic criteria. In addition, this paper highlight some gaps in the $\mathrm{MOH}$ criteria, such as the absence of definition of overuse and misuse, and the need for withdrawal syndrome criteria related to $\mathrm{MOH}$ non-opioids medications. Clinical trials are needed to overcome the failures of the current criteria.

Author's contribution: TACL -Conception or design of the work; Data collection; Data analysis and interpretation; Drafting the article; Critical revision of the article; Final approval of the version to be published. MFPP - Conception or design of the work; Data collection; Data analysis and interpretation; Drafting the article; Critical revision of the article; Final approval of the version to be published. SDS - Drafting the article; Critical revision of the article; Final approval of the version to be published.

Conflict of interest: The authors declare that there is no conflict of interest.

Funding: The authors declare that there is no funding 
Thaiza Agostini Córdoba de Lima

https://orcid.org/0000-0001-6694-5259,

Mario Fernando Prieto Peres

https://orcid.org/0000-0002-0068-1905

Stephen D Silberstein

https://orcid.org/0000-0001-9467-5567

\section{References}

1. Diener HC, Dodick D, Evers S, Holle D, Jensen RH, Lipton RB, . . . Schwedt T. Pathophysiology, prevention, and treatment of medication overuse headache. Lancet Neurol 2019;18(9):891-902 Doi:10.1016/s14744422(19)30146-2

2. The International Classification of Headache Disorders, 3rd edition (beta version). Cephalalgia 2013;33(9):629808 Doi: $10.1177 / 0333102413485658$

3. Vincent $M B$. Controversy over the classification of medication-overuse headache. Curr Pain Headache Rep 2012;16(1):80-85 Doi:10.1007/s $11916-011$ 1-0229-8

4. Scher Al, Rizzoli PB and Loder EW. Medication overuse headache: An entrenched idea in need of scrutiny. Neurology 2017;89(12):1296-1304 Doi:10.1212/ wnl.0000000000004371

5. Bigal ME, Serrano D, Buse D, Scher A, Stewart WF and Lipton RB. Acute migraine medications and evolution from episodic to chronic migraine: a longitudinal population-based study. Headache 2008;48(8): 1157 1168 Doi: $10.1111 /$ j.1526-4610.2008.01217.x

6. Carlsen LN, Munksgaard SB, Jensen RH and Bendtsen L. Complete detoxification is the most effective treatment of medication-overuse headache: A randomized controlled open-label trial. Cephalalgia 2018;38(2):225-236 Doi: $10.1177 / 0333102417737779$

7. Valguarnera $F$ and Tanganelli P. The efficacy of withdrawal therapy in subjects with chronic daily headache and medication overuse following prophylaxis with topiramate and amitriptyline. Neurol Sci 2010;31(Suppl 1):175-177 Doi:10.1007/s10072010-0319-0

8. Paolucci M, Altamura C, Brunelli N, Rizzo AC, Assenza F, Pasqualetti $\mathrm{P}$ and Vernieri F. Methylprednisolone plus diazepam i.v. as bridge therapy for medication overuse headache. Neurol Sci 2017;38(11):2025-2029 Doi:10.1007/s10072-017-3098-z

9. Smith SM, Dart RC, Katz NP, Paillard F, Adams EH, Comer SD, . . . Dworkin RH. Classification and definition of misuse, abuse, and related events in clinical trials: ACTTION systematic review and recommendations. Pain 2013;154(11):2287-2296 Doi:10.1016/j. pain.2013.05.053

10. Cooper R. Diagnostic and Statistical Manual of Mental
Disorders (DSM). KO Knowledge Organization 2017;44(8):668-676 Doi:10.5771/0943-7444-2017. 8-668

11. Radat F, Creac'h C, Guegan-Massardier E, Mick G, Guy N, Fabre N, . . . Lantéri-Minet M. Behavioral dependence in patients with medication overuse headache: a cross-sectional study in consulting patients using the DSM-IV criteria. Headache 2008;48(7): 1026 1036 Doi: $10.1111 /$ j.1526-4610.2007.00999.x

12. Lundqvist C, Gossop M, Russell MB, Straand $J$ and Kristoffersen ES. Severity of Analgesic Dependence and Medication-overuse Headache. J Addict Med 2019;13(5):346-353 Doi:10.1097/ adm.0000000000000504

13. Hasin D, Hatzenbuehler ML, Keyes K and Ogburn E. Substance use disorders: Diagnostic and Statistical Manual of Mental Disorders, fourth edition (DSMIV) and International Classification of Diseases, tenth edition (ICD-10). Addiction 2006;101(Suppl 1):59-75 Doi:10.1111/i.1360-0443.2006.01584.x

14. Radat F, Chanraud S, Di Scala G, Dousset V and Allard $M$. Psychological and neuropsychological correlates of dependence-related behaviour in medication overuse headaches: a one year follow-up study. I Headache Pain 2013; 14(1):59 Doi:10.1186/11 29-2377-14-59

15. Figee $M$, Pattii T, Willuhn I, Luigies J, van den Brink W, Goudriaan A, ... Denys D. Compulsivity in obsessive-compulsive disorder and addictions. Eur Neuropsychopharmacol 2016;26(5):856-868 Doi:10.1016/i.euroneuro.2015.12.003

16. Shagbazyan A.E., Sergeev A.V., Evdokimova E.M., Kurbanbagamaeva R.R. and G.R T. The role of emotional and behavioral disorders in the development of drug dependence in patients with medication-induced headache (review). Nevrologiya, Neiropsikhiatriya, Psikhosomatika 2016;8(3):76-81 Doi:10.14412/2074271 1-2016-3-76-81

17. Chanraud S, Di Scala G, Dilharreguy B, Schoenen J, Allard $M$ and Radat F. Brain functional connectivity and morphology changes in medicationoveruse headache: Clue for dependence-related processes? Cephalalgia 2014;34(8):605-615 Doi:10.1177/0333102413519514

18. Radat $F$ and Lanteri-Minet M. What is the role of dependence-related behavior in medication-overuse headache? Headache 2010;50(10):1597-1611 Doi:10.1111/j.1526-4610.2010.01755.x

19. Ferrari A, Coccia C and Sternieri E. Past, present, and future prospects of medication-overuse headache classification. Headache 2008;48(7):1096-1102 Doi:10.1111/j.1526-4610.2008.00919.x

20. Roerig JL, Steffen KJ, Mitchell JE and Zunker C. Laxative 
abuse: epidemiology, diagnosis and management. Drugs 2010;70(12):1487-1503 Doi:10.2165/11898640000000000-00000

21. CDC. Prescription Painkiller Overdoses: A Growing Epidemic, Especially Among Women: Vital Signs; 2012 Available from: https://www.cdc.gov/vitalsigns/ prescriptionpainkilleroverdoses/index.html.

22. Bell K and Salmon A. Pain, physical dependence and pseudoaddiction: redefining addiction for 'nice' people? Int J Drug Policy 2009;20(2):170-178 Doi:10.1016/i. drugpo.2008.06.002

23. Cooper R. Over-the-counter medicine abuse-a review of the literature. J Subst Use 2013;18(2):82-107 Doi:10.3 $109 / 14659891.2011 .615002$
24. Heit HA. Addiction, physical dependence, and tolerance: precise definitions to help clinicians evaluate and treat chronic pain patients. J Pain Palliat Care Pharmacother 2003; 17(1):15-29 Doi:10.1080/j354v17n01_03

25. National Institute on Drug Abuse. Drugs, brains, and behavior: The science of addiction. 1 ed Nida; 2010. 29p.

26. O'Brien CP, Volkow $N$ and Li TK. What's in a word? Addiction versus dependence in DSM-V. Am J Psychiatry 2006;163(5):764-765 Doi:10.1176/ ajp.2006.163.5.764

27. Weaver $M$ and Schnoll $S$. Abuse liability in opioid therapy for pain treatment in patients with an addiction history. Clin J Pain 2002;18(4 Suppl):61-69 Doi:10.1097/00002508-200207001-00007 